BULLETIN OF THE

AMERICAN MATHEMATICAL SOCIETY

Volume 79, Number 2, March 1973

\title{
WEIGHTED APPROXIMATION FOR MODULES OF CONTINUOUS FUNCTIONS
}

\author{
BY W. H. SUMMERS ${ }^{1}$ \\ Communicated by François Treves, September 25, 1972
}

Nachbin [6] has enjoyed notable success with the weighted approximation problem in both the real and selfadjoint complex cases, but little progress has yet been realized in the general complex case, even though interest dates from the problem's formative stages (cf. [4, p. 1057], [5, p. 126]). The purpose of the present note is to provide an answer to this question in a setting which occupies a pivotal position in the theory developed by Nachbin; namely, the bounded case of the weighted approximation problem.

1. Preliminaries. In what follows, all functions will be assumed complex valued unless explicitly stated otherwise, while $X$ will denote a completely regular Hausdorff space, $C(X)$ will denote the algebra of all continuous functions on $X$, and $B_{0}(X)$ will denote the algebra of all bounded functions on $X$ which also vanish at infinity. We will assume that $B_{0}(X)$ is equipped with the uniform (convergence) topology induced by $\|\cdot\|$, the usual supremum norm defined on the bounded functions on $X$.

We now introduce a set $V$ of nonnegative upper semicontinuous functions on $X$; the elements of $V$ being referred to as weights. The corresponding weighted space $C V_{0}(X)$ is the locally convex topological vector space obtained by equipping the linear subspace consisting of those $f \in C(X)$ such that $f v \in B_{0}(X)$ for every $v \in V$ with the weighted topology $\omega_{V}$ generated by the seminorms $p_{v}$, one for each $v \in V$, defined on this space by $p_{v}(f)=$ $\|f v\|$. Since there is no loss of generality in so doing, we will assume that if $u, v \in V$ and $\lambda \geqq 0$, then there is a $w \in V$ for which $\lambda u, \lambda v \leqq w$ (pointwise); i.e., $V$ is a Nachbin family on $X[8$, p. 90]. In addition, we henceforth assume that a subalgebra $A$ of $C(X)$ and a linear subspace $W$ of $C V_{0}(X)$, where $W$ is an $A$-module with respect to pointwise multiplication, have been specified. The weighted approximation problem [6, p. 293] asks for a description of the closure of $W$ in $C V_{0}(X)$.

2. The bounded case of the weighted approximation problem. The setting Nachbin termed the bounded case of the weighted approximation problem $[6$, p. 294] is the one in which every $a \in A$ is bounded on the support of each $v \in V$, and it is in this situation that a meaningful characterization of

AMS(MOS) subject classifications (1970). Primary 41A65, 46E10.

${ }^{1}$ This research was supported in part by National Science Foundation Grant GP-34370. 
$\operatorname{cl}(W)$ can now be given without placing any additional restrictions on $A$. The description we will give arises from a localization of the weighted approximation problem: denoting the collection of maximal antisymmetric sets for $A$ by $\mathscr{K}_{A}$, we say that a function $f \in C V_{0}(X)$ is in $\mathrm{cl}_{A}(W)$, the $A$-local closure of $W$ in $C V_{0}(X)\left[8\right.$, p. 91], if, for any $K \in \mathscr{K}_{A}$ and $v \in V$, there exists a $w \in W$ such that $\sup \{|f(x)-w(x)| v(x): x \in K\} \leqq 1$. Clearly, $\mathrm{cl}_{A}(W)$ is a closed linear subspace of $C V_{0}(X)$ which also contains $W$.

2.1. THEOREM. In the bounded case of the weighted approximation problem, the closure of $W$ in $C V_{0}(X)$ always coincides with $\mathrm{cl}_{A}(W)$.

Before outlining a proof of this theorem, we indicate some of its immediate consequences.

2.2 Corollary (Nachbin [6, p. 295]). If $A$ is selfadjoint, then $W$ is localizable under $A$ in the bounded case of the weighted approximation problem.

Theorem 2.1 not only contains Nachbin's theorem as a special case, but it can also be regarded as an extension of Bishop's generalized StoneWeierstrass theorem [1] to the class of weighted spaces under discussion. Indeed, as indicated in the earlier paper [8] where this theorem was first conjectured, appropriate choices of $X$ and $V$ yield the setting for both Bishop's theorem and Glicksberg's analogous result [3] for the strict topology. Moreover, the version of Bishop's theorem recently obtained by Prolla $[7$, p. 284] and many noteworthy variations of the classical Stone-Weierstrass theorem (see [8, p. 97]) are all subsumed by the above theorem.

3. Proof of Theorem 2.1: an outline. As did Glicksberg [3] and Prolla [7] before us, we employ a modification of de Branges' elegant proof of the Stone-Weierstrass theorem [2]. However, the key to our argument is in avoiding the need for the characterization of the equicontinuous subsets of $C V_{0}(X)^{*}$, the topological dual of $C V_{0}(X)$, on which the aforementioned proofs so strongly depend.

For each $v \in V$, we let $\mathscr{S}_{v}=\left\{g v: g \in C V_{0}(X)\right\}$. These linear subspaces of $B_{0}(X)$ lead to a notion of "support" for elements of $B_{0}(X)^{*}$.

3.1. Lemma. Assume $T \in B_{0}(X)^{*}$. Corresponding to each $v \in V$, there is a set $S_{v}(T)$ contained in $X$ which satisfies the following properties:

(1) $S_{v}(T) \subseteq \operatorname{spt} v$;

(2) if $h \in \mathscr{S}_{v}$ is such that $h(x)=0$ for all $x \in S_{v}(T)$, then $T h=0$;

(3) if there exists $h \in \mathscr{S}_{v}$ such that $T h \neq 0$, then $S_{v}(T)$ is not empty;

(4) if $T \geqq 0$, then $T h>0$ whenever $h \in \mathscr{S}_{v}, h \geqq 0$, and $h(x)>0$ for at least one $x \in S_{v}(T)$. 
3.2. Lemma. Assume $T$ is an extremal point of $(W v)^{0} \cap B_{1}^{0}$, where $v \in V$ and $B_{1}=\left\{h \in B_{0}(X):\|h\| \leqq 1\right\}$. In the bounded case of the weighted approximation problem, $S_{v}(T)$ is an antisymmetric set for $A$.

Given the preceding lemmas, de Branges' technique can be applied to obtain the following, and final, step in the proof of Theorem 2.1.

3.3. LEMMA. In the bounded case of the weighted approximation problem, given $v \in V$, the uniform closure in $B_{0}(X)$ of $W v$ always contains $\mathrm{cl}_{A}(W) v$.

\section{REFERENCES}

1. E. Bishop, A generalization of the Stone-Weierstrass theorem, Pacific J. Math. 11 (1961), 777-783. MR 24 \# A3502.

2. L. de Branges, The Stone-Weierstrass theorem, Proc. Amer. Math. Soc. 10 (1959), 822-824. MR 22 \# 3970.

3. I. Glicksberg, Bishop's generalized Stone-Weierstrass theorem for the strict topology, Proc. Amer. Math. Soc. 14 (1963), 329-333. MR 26 \#4165.

4. L. Nachbin, On the weighted polynomial approximation in a locally compact space, Proc. Nat. Acad. Sci. U.S.A. 47 (1961), 1055-1057. MR 27 \# 513.

5. — Weighted approximation over topological spaces and the Bernstein problem over finite dimensional vector spaces, Topology 3 (1964), suppl. 1, 125-130. MR 28 \# 4286.

6. - Weighted approximation for algebras and modules of continuous functions: real and self-adjoint complex cases, Ann. of Math. (2) 81 (1965), 289-302. MR 31 \#628.

7. J. B. Prolla, Bishop's generalized Stone-Weierstrass theorem for weighted spaces, Math. Ann. 191 (1971), 283-289.

8. W. H. Summers, The general complex bounded case of the strict weighted approximation problem, Math. Ann. 192 (1971), 90-98.

Department of Mathematics, University of Arkansas, Fayetteville, Arkansas 72701 